\title{
Decentralized Distributed Deep Learning in Heterogeneous WAN Environments
}

\author{
Extended Abstract
}

\author{
Rankyung Hong \\ University of Minnesota \\ hongx293@umn.edu
}

Motivation: Large volumes of customer-related data and logs are increasingly generated in a geo-distributed manner, e.g., by users and edge devices distributed geographically. There is a growing interest to analyze this data and provide insightful user experiences through cloud-based deep learning (DL) products such as Amazon Sagemaker, Rekognition, etc. Much of this learning currently takes place on centralized high-performance cloud infrastructures such as GPU clusters. However, due to concerns of efficiency, cost, and privacy, such DL may be better carried out across a combination of edge servers, private clouds, and public clouds. Instead of moving all the data to a public cloud across the WAN, it would be desirable to train some of the customer-generated data at the edges or private cloud along with public cloud. Therefore, it is inevitable that distributed deep learning in WANs is used for training DL models using geo-distributed machines with heterogeneous computing and network capabilities.

Distributed deep learning training in heterogeneous WANs is challenging for the following reasons. Distributed DL training typically requires exchanging large number of parameters among multiple machines. WAN bandwidth is highly scarce compared to the size of parameters. This bandwidth constraint can cause significant drop in model accuracy during asynchronous training and inefficient resource use during synchronous training in distributed DL. In addition, in a WAN-based heterogeneous network comprising cloud servers and edges servers, with large gaps in their computing resources and network capacity, the training can slow down significantly or even fail to converge. Thus, the key problem is how to scale the size of parameters shared over WANs and how to mitigate the disparities in training speed among heterogeneous machines. Proposed Solution: We propose a decentralized distributed deep learning framework for such heterogeneous WAN-based infrastructures. The framework dynamically and automatically adjusts 1) the frequency of parameter sharing, 2) the size of parameters shared depending on individual network bandwidth and data processing power, and 3) introduces a new scaling factor to control the degree of contribution to parameter updates by considering the amount of data trained during unit time in each device. In addition, the framework takes cloud resource cost and target accuracy into account to tune the three system parameters above.

Permission to make digital or hard copies of part or all of this work for personal or classroom use is granted without fee provided that copies are not made or distributed for profit or commercial advantage and that copies bear this notice and the full citation on the first page. Copyrights for third-party components of this work must be honored.

For all other uses, contact the owner/author(s).

SoCC '18, October 11-13, 2018, Carlsbad, CA, USA

(C) 2018 Copyright held by the owner/author(s).

ACM ISBN 978-1-4503-6011-1/18/10 ..\$15.00

https://doi.org/10.1145/3267809.3275447

\author{
Abhishek Chandra \\ University of Minnesota \\ chandra@umn.edu
}

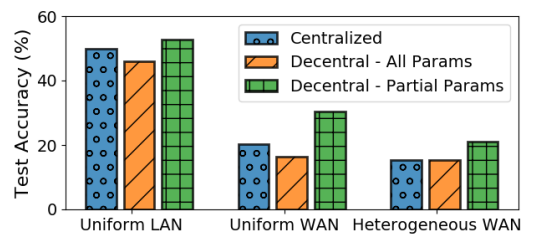

Figure 1: Test accuracy of DL models trained in various network environments and parameter sizes; Decentralized DL with partial parameter sharing outperforms.

There is some recent work on distributed DL training in WANs like Gaia [2] and LANs like Ako [4]. They reduce network communication cost by sharing partial parameters, but do not consider heterogeneous networks including public and private clouds, and edge servers. However, decentralized DL training without parameter servers proposed by Ako naturally inherits the concept of loosely coupled DL training, which is more feasible for our target environment.

Implementation and Preliminary Results: We have implemented $^{1,2}$ Ako algorithm in TensorFlow [1] to examine its performance in various environments with CIFAR10 [3]. We measured test accuracies of a DL model trained for 300 seconds in uniform and heterogeneous LANs and WANs. The preliminary results in figure 1 show that sharing small size of parameters (partial params) is more effective to increase the accuracy faster when machines are highly network bandwidth-constrained during training.

However, the size of parameters shared every iteration is fixed across machines in the existing work since they do not consider heterogeneous networks. Moreover the data size trained in unit time can vary depending on computing power. Thus, it is important that the frequency, size of parameters as well as its contribution to parameter updates should be more precisely and dynamically controlled by the DL framework based on different individual system resources and environments for better model accuracy.

Acknowledgement. This work is supported in part by NSF grant III-1422802.

\section{REFERENCES}

[1] M. Abadi et al. Tensorflow: a system for large-scale machine learning. In OSDI, volume 16, pages 265-283, 2016.

[2] K. Hsieh et al. Gaia: Geo-distributed machine learning approaching lan speeds. In NSDI, pages 629-647, 2017.

[3] A. Krizhevsky and G. Hinton. Learning multiple layers of features from tiny images. Technical report, Citeseer, 2009.

[4] P. Watcharapichat et al. Ako: Decentralised deep learning with partial gradient exchange. In Proceedings of the Seventh ACM Symposium on Cloud Computing, pages 84-97. ACM, 2016

\footnotetext{
${ }^{1}$ Source code: https://github.com/mesh-umn/TF.AKO

${ }^{2}$ https://www-users.cs.umn.edu/ chandra/tfako/home.html
} 